\title{
A literature review on the optimization of legged robots
}

\author{
Manuel Fernando Silva and JA Tenreiro Machado
}

\begin{abstract}
Over the last two decades the research and development of legged locomotion robots has grown steadily. Legged systems present major advantages when compared with 'traditional' vehicles, because they allow locomotion in inaccessible terrain to vehicles with wheels and tracks. However, the robustness of legged robots, and especially their energy consumption, among other aspects, still lag behind mechanisms that use wheels and tracks. Therefore, in the present state of development, there are several aspects that need to be improved and optimized. Keeping these ideas in mind, this paper presents the review of the literature of different methods adopted for the optimization of the structure and locomotion gaits of walking robots. Among the distinct possible strategies often used for these tasks are referred approaches such as the mimicking of biological animals, the use of evolutionary schemes to find the optimal parameters and structures, the adoption of sound mechanical design rules, and the optimization of power-based indexes.
\end{abstract}

Keywords

Artificial legged locomotion, genetic algorithms, locomotion gaits, optimization, walking robots

\section{Introduction}

Legged robots present significant advantages over traditional vehicles with wheels and tracks. Wheeled vehicles demand paved surfaces (or at least regular) in order to move, being extremely fast and effective in them. At the same time these mechanisms can be simple and lightweight. However, more than $50 \%$ of the earth's surface is inaccessible to traditional vehicles, it being difficult, or even impossible, for wheeled vehicles to deal with large obstacles and surface unevenness. Even all-terrain vehicles can only surpass small obstacles and surface unevenness, but at the cost of high energy consumption (Bekker, 1960).

Regarding tracked vehicles, although they present increased mobility in difficult terrains, they are not able to surpass many of the difficulties and their energy consumption is relatively high. To these problems, one must add the fact that traditional vehicles leave continuous ruts on the ground, which in some situations is disadvantageous as, for instance, from the environmental point of view.

From what was seen, it is possible to conclude that leggedlocomotion systems presentasuperior mobility in natural terrains, since these vehicles may use discrete footholds for each foot, in opposition to wheeled vehicles, that need a continuous support surface. Therefore, these vehicles may move in irregular terrains, by varying their legs configuration, in order to adapt themselves to surface irregularities and, furthermore, the feet may establish contact with the ground in selected points in accordance with the terrain conditions. For these reasons, legs are inherently adequate systems for locomotion in irregular ground. When the vehicles move in soft surfaces, as for instance in sandy soil, the ability to use discrete footholds in the ground can also improve the energy consumption, since they deform the terrain less than wheeled or tracked vehicles. Therefore, the energy needed to get out of the depressions is lower 
(Bekker, 1960,1969) and the contactarea among the foot and the ground can be made in such a way that the ground support pressure can be small. Moreover, the use of multiple degrees-of-freedom (DOF) in the leg joints, allows legged vehicles to change their heading without slippage. It is also possible to vary the body height, introducing a damping and decoupling effect between terrain irregularities and the vehicle body (and as a consequence of its payload). In what concerns locomotion, it should also be mentioned the possibility that these systems present to hugging themselves to the terrain in which they move. This is particularly true, in case they move, for instance, over the outside surface of pipes, in order to increase their balance ability (Kaneko et al., 2002).

Although legged vehicles present all these potential advantages, in the current state of development, there are several aspects that have to be improved and optimized.

With this idea in mind, different optimization strategies have been proposed and applied to these systems, either during their design and construction, or during their operation, namely in what concerns to the selection of the gait to be adopted (Alexander, 1984; Hornby et al., 2000; Silva and Machado, 2005; Stelzer et al., 2003) and its adaptation to the terrain and locomotion conditions (Estremera et al., 2003; Weingarten et al., 2004). Among the optimization criteria followed by distinctauthors one mayincludeaspects related to energy efficiency (Buehler, 2001; Silva and Machado, 2005; Wang et al., 2008), stability (Albert and Gerth, 2001; Garcia et al., 2002; Hardt and von Stryk, 2002), velocity (Huang and Waldron, 1990; Hugel and Blazevic, 1999; Hugel et al., 2000; Kohl and Stone, 2004), comfort (Neuhaus and Kazerooni, 2000), mobility (Hugel and Blazevic, 1999; Quinn et al., 2001; Warren, 2002) and environmental impact (Ihme and Deutscher, 2001).

This paper reviews several approaches that have been adopted for the optimization of the mechanical structure and the locomotion modes of artificial legged systems. Among them are referred the mechatronic mimic of the characteristics of biological animals (Albiez and Berns, 2005; Alexander, 2005; Breithaupt et al., 2002; Hennion et al., 2005; Quinn et al., 2001), the use of genetic algorithms (GA) for the optimization of the legged structure parameters (Reyes and Gonzalez, 2005; Ziegler and Banzhaf, 2001), the adoption of sound mechanical design rules (Yoneda, 2001), or the optimization of power/energy-based indices (Silva and Machado, 2005).

The remainder of this paper is organized as follows. Section two presents some biological approximations to walking machines and section three introduces the adoption of evolutionary algorithms for designing legged robots. Sections four and five present mechanical design rules and optimizing indices for the optimization of artificial walking robots, respectively. Section six discusses other approaches to the problem under analysis. Finally, section seven presents the main conclusions of this study.

\section{Biological approximations}

Since legged robots are inspired in animals observed in nature, a frequent approach for their design and construction is to develop a mechatronic mimic of the animal that is intended to replicate, either in terms of its physical dimensions, or in terms of characteristics such as the gait and the actuation of the limbs. With this objective in mind, detailed studies of the locomotion and anatomy of the animals have been made. Works joining researchers from the robotics and the biology areas are often presented (Laksanacharoen et al., 2000; Schneider et al., 2005; Witte, et al., $2001 \mathrm{a}, \mathrm{b})$. Several examples of robots that have been developed based on this approximation are discussed in Silva and Machado (2007), for example, the Lobster Robot, that intends to be a lobster mimic (Ayers, 2004), the CWRU Robot II (Espenschied et al., 1996) that represents a stick-insect and the CWRU Robot III (Nelson and Quinn, 1998; Nelson et al., 1997) that intends to mimic (17:1 scale) the Blaberus Discoidalis cockroach.

This approach is also followed in the development of biped and humanoid robots. The designers of these systems get much of their inspiration from mankind, as proved by several machines with characteristics similar to those of humans, namely in the number of DOF and in their dimensions. Among the large number of examples adopting this approach one can

mention the following robots:

- the WABIAN humanoid, whose size and joint range of motion is based on an adult human (Yamaguchi and Takanishi, 1998);

- the biped developed by Caldwell et al. (1997) to test the actuation using artificial pneumatic muscles, that presents anthropomorphic dimensions;

- the BIP robot that presents the kinematic and dynamic parameters close to the anthropomorphic values of an human, with $1.70 \mathrm{~m}$ height and $90 \mathrm{~kg}$ mass (Espiau et al., 1997);

0 the Honda Humanoid Robot model P2 that, on an initially phase of development, presented the dimensions, joint locations, ranges of motion and centre of gravity equivalent to the human leg (Hirai etal., 1998). Latter, it was verified that was difficult to satisfy all the conditions, and some simplifications were made;

- the BIP2000 robot, with kinematic and dynamic parameters close to the values of an human, with $1.80 \mathrm{~m}$ height (Espiau and Sardain, 2000); 
- the Robian II, with dimensions and weights approximate to the average European man (Gravez et al., 2002);

- the biped robot Johnnie, that presents a geometry corresponding to that of a male human with a body height of $1.8 \mathrm{~m}$ (Lohmeier et al., 2004).

The main problem that can be pointed to the biomimetic approach concerns the resulting mechanisms. In fact, mechanisms that are a straight copy of the joints and links of biological animals tend to present a large number of DOF, with the corresponding difficulties in the control algorithms. Moreover, the mechanical complexity is one of the largest sources of mal-functions and considerably increases cost and weight.

To overcome these difficulties, some authors have proposed the adoption of walking robots with reduced number of DOF and reduced actuation. This approach is based on the development of robots that are somehow different from their biological counterparts keeping, however, the concept of legs for the locomotion. This development line supports itself on the mechanical simplicity and, therefore, promotes robustness. Among the diverse examples of robots built according to this paradigm, one can refer the quadrupeds Hyperion (Yoneda, 2001), SCOUT-I (Buehler et al., 1998, 1999), and SCOUT-II (Hawker and Buehler, 2000; Papadopoulos and Buehler, 2000), the hexapod Sprawlita (Cham et al., 2002), and the quadrupeds with Whegs, RHex (Saranli et al., 2001) and Whegs I (Quinn et al., 2002).

\section{Evolutionary strategies}

Evolutionary strategies are an alternative way of imitating nature. The characteristics of animals are not directly copied but, instead, the process that nature conceives for its generation and evolution is replicated. One possibility to implement this idea makes use of $\mathrm{GA}$ as the engine to generate robot structures (Farritor et al., 1996; Leger, 2000; Nolfi and Floreano, 2000; Pires et al., 2001). A modular approach to the design is performed in these applications. There is a library of elementary components, such as actuated joints, links, gears, power supplies, amongst others.

Several of these elements are combined in order to originate different structures. The generated structures are evaluated, using pre-defined fitness functions, and recombined among them using genetic operators. Finally, the selection process originates a robotic system that represents the best design for a specific application.

These computer applications present the capability of an easy reconfiguration and application in the generation of robotic systems for very distinct situations (Farritor, et al., 1996; Leger, 2000). In the literature there are also works on which evolutionary strategies are adopted to generate the structure of a specific robot.

Jua' rez-Guerrero et al. (1998) developed a biped robot using evolutionary strategies. The final goal was to evolve the biped robot structure, equipped with a passive tail to help keeping balance. The robot struc- ture should be able to implement a simple gait and to fulfil a set of restrictions, namely: minimum and max- imum dimensions, maximum motor torque, step length (lower than $0.30 \mathrm{~m}$ ), maximum foot elevation (lower than $0.05 \mathrm{~m}$ ) and maximum robot weight (lower than $30 \mathrm{~kg}$ ). The attained robot was built and its adequacy to the proposed task was verified. Besides the locomotion mechanism, the process followed by these authors also allowed the optimization of the distance between the robot centre of mass to the tail, the tail length and the foot surface.

The use of GA for optimizing the structure of a biped robot was also adopted by Ishiguro et al. (2002). In their study, the robot was able to move passively, on sloped surfaces, and through actuated joints, in flat surfaces. In a first phase, the robot body parameters (for example, length and body mass of each body part) were optimized using a GA and assuming a passive robot. These authors considered for fitness function the distance travelled by the robot and the number of steps taken, during a 20 sec downhill locomotion, subject to the restriction that the height of the waist could not fall beyond $70 \%$ of the height of the upright posture.

After optimizing the robot structure (the developed structure was able to be implemented with passive dynamic walking), these authors made use of a second GA to optimize the parameters of a controller based on a Central Pattern Generator (CPG) scheme. In this second GA, the fitness function was designed in such a way that an individual receives higher scores when it travels a longer distance with less energy consumption. The obtained results have shown that passive dynamic walkers provide significantly high evolvability compared to other embodiments thatcan not perform passive dynamic walking. These results lead to the conclusion that embodiments showing passive dynamic walking can remarkably increase the efficiency of developing controllers. Furthermore, although the size of the search space is larger in the case of coupled evolution of morphology and control, the evolutionary runs that were conducted significantly outperform others in which merely the biped controller is evolved (Ishiguro et al., 2002).

Contrary to the examples described previously, where the structure and the control system are optimized separately, Lipson and Pollack (2000) proposed 
the use of GA for the simultaneous generation of the mechanical structure and the robot controller. Given the task of locomotion, they apply these ideas to evolve distinct robots, with different mechanics and control, but that ultimately fulfil the desired objective. As the robot's building blocks, are used linear actuators and bars for the morphology, and sigmoidal neurons for the control. The fitness function was defined as the net Euclidean distance that the center-of-mass of an individual has moved over a fixed number (1224) of cycles of its neural control. These authors performed several runs of the GA, for the task of locomotion, and the evolved robots exhibited various methods of locomotion, including crawling, ratcheting and some form of pedalism. The emerged solution has the particularity that the robots are manufactured through rapid prototyping methods and can be recycled after fulfilling their mission (Lipson and Pollack, 2000). Hornby et al. (2001) further developed these ideas, and constructed an actual robot from an evolved design.

Endo et al. (2002a, b) also considered a GA to optimize simultaneously the structure and the control system of the biped humanoid robot PINO. In order to start the optimization process (i.e., the evolution of the robot structure) they used a model based on a multi-link structure. The result of the robot structure evolution was, in a first phase, the optimum length of the links (Endo et al., 2002b) and, in a second phase, the optimum positioning and orientation of the servomotors (Endo et al., 2002a). Regarding the control system, they studied two different architectures, namely (i) a neural network and (ii) neural oscillators. The GA is multi-objective and is implemented in two phases. In a first phase, the fitness function is based in the distance travelled by the robot. The best 20 robots found in this phase are the initial population of the second phase where are optimized fitness functions based on the energy efficiency and the stability of the robot locomotion (Endo et al., 2003).

The main criticism to the design approach based in evolutionary strategies lays in its convergence. In fact, there is some uncertainty about achieving a solution, due to the high complexity needed for the robot to be of practical use. As an example of a work that is being implemented one can mention the robot developed by Endo and Maeno (2003).

The techniques of evolutionary programming have proven useful in the optimization of legged robots, given the relative high number of parameters presented by them (and that may be the object of optimization). However, until now only a reduced number of prototypes has been built according to the results given by the studies developed so far. Furthermore, there is no commonly accepted solution (an optimum design) for a walking robot that has been the result of such a research approach and no study has yet been developed to compare the different designs proposed by distinct researchers.

\section{Mechanical project}

The approaches to the systems design discussed in the two previous sections are inspired in the strategies found in nature. However, it is important to keep in mind that legged robots are machines. Therefore, the first aspect to consider in their design phase should be the adequate implementation from the mechanical and physical viewpoints.

In this line of thought, Habumuremyi and Doroftei (2001) compiled the characteristics of several structures that can be adopted for the legs of artificial locomotion systems.

Hirose and Arikawa (2000) examined several concepts to be adopted during the design of legged vehicles. The main idea is to maximize the power developed in the system (concept of 'coupled actuation') and to maximize the energy efficiency (concept of 'actuator gravitational decoupling'). The technique of actuator gravitational decoupling was adopted in several robots (Genta and Amati, 2001; Koyachi et al., 1991; Senta et al., 1995) and can be implemented not only during the system design, but also in the posture during locomotion (Hirose and Arikawa, 2000).

In some cases, for designing a robot, empirical knowledge of mechanics and physics is supported as an adopted approach. The design of the equipments has the objective of minimizing some situation penalizing the performance of the robot under consideration (Hirose et al., 1997; Yamaguchi and Takanishi, 1998).

Another method for the optimization of the robot structure based on biology research (Alexander, 1990), considers legs equipped with actuators introducing joint compliance. In this way, it is possible to store and to release the kinetic and the potential energies of the robot legs and body, during the different phases of the locomotion cycle. Raby and Orin (1999) make use of this approach with a passive hexapod robot (Figure 1). The proposed robot has legs with two DOF, one rotational at the hip and one prismatic at the knee, having each joint a spring to allow some compliance. After optimizing the locomotion parameters, they conclude that is required a small amount of energy to keep the robot in the periodic locomotion.

\section{Optimization of power/energy based indices}

Concerning the weakness of artificial locomotion systems, one of the most serious problems faced by legged 


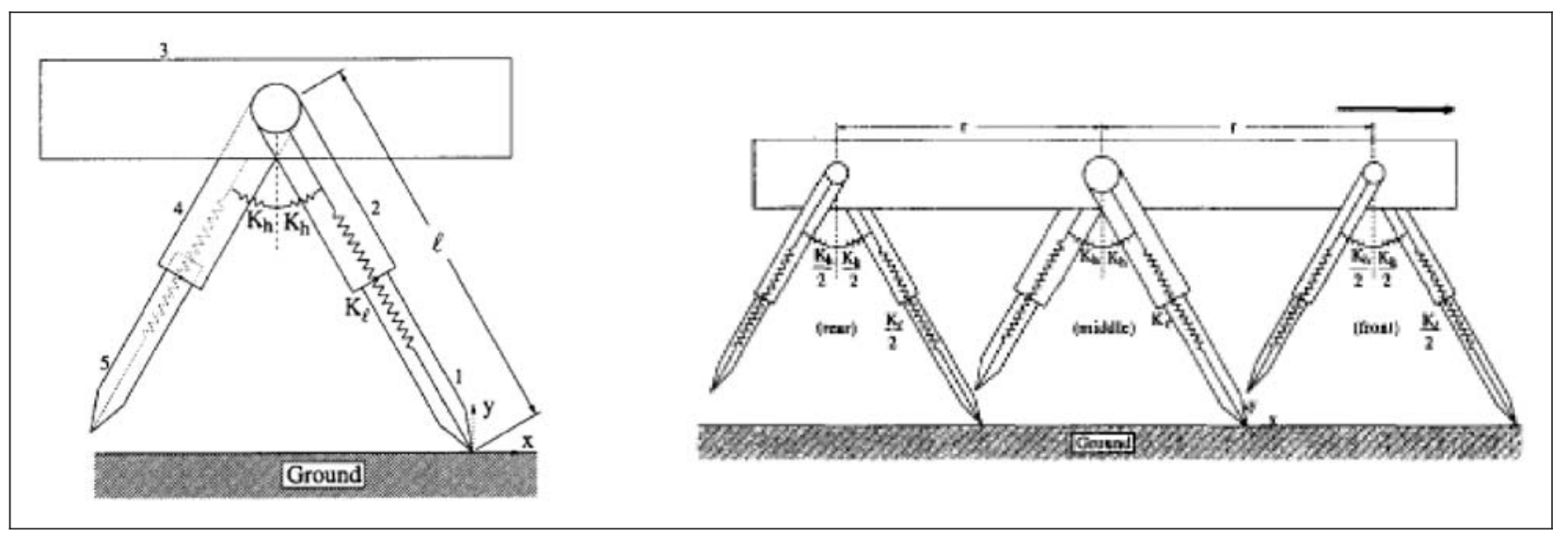

Figure 1. Biped model with leg and hip compliance (left) and hexapod model with compliant legs (right). Reproduced with kind permission from the IEEE (Raby and Orin, 1999).

robot designers is the optimization of the energy consumption.

It is verified that the locomotion efficiency of the existing robots has been very low when compared either with the living animals, or with the locomotion through wheels. As an example, Kajita et al. (2002) performed a simulation of the HRP1 humanoid robot running gait and concluded that the robot would need actuators from 28 up to 56 times more powerful than those available in the real humanoid. Besides this result, they also estimated that the energy consumption would be about ten times higher than that of a human adopting the same gait.

It is expected that in the future the locomotion through artificial legs will become one of the most efficient transportation modes from the energy viewpoint. Therefore, a deeper attention should be devoted to this aspect in the development of such systems, namely through the use of power or energy-based optimization criteria during the design, construction and exploration stages, since this influences the choice of the mechanical structure, the type of propulsion and the power supply system.

There is an important hypothesis that seems to explain most of the legs and body motions during human locomotion. This hypothesis states that, during locomotion, humans control their muscles activity, moving the various body segments, in such a way that the metabolic energy needed to travel a certain distance is minimized (Neuhaus and Kazerooni, 2000). It is also recognized that most legged locomotion animals optimize the energy efficiency in detriment to the motion smoothness (Alexander, 1984; Dunn and Howe, 1996). Based on these ideas, several authors analyzed the optimization of locomotion structures, and their gaits, based on energy criteria.

An index used frequently to analyze and compare the performance of legged robots is the specific resistance $(p)$.
This index, originally introduced by Gabrielli and Von Ka' rma' n (1950) to compare the locomotion efficiency of different sorts of vehicles (Figure 2), is defined as:

$$
\rho=\frac{P}{M g V_{\max }}
$$

where $P$ is the vehicle maximum power, $M$ is its mass, $g$ is the gravitational acceleration and $V_{\max }$ is the maximum velocity that it can reach. The specific resistance can alternatively be defined by (De Lasa and Buehler, 2001):

$$
\rho\left(V_{F}\right)=\frac{P\left(V_{F}\right)}{M g V_{F}}
$$

as a function of the travelling velocity $V_{F}$.

This index was adopted to study the performance of a passive biped robot after introducing compliance into its joints (Van der Linde, 1998). It was also used to compare the performance of the RHex hexapod robot when crossing obstacles (Saranli et al., 2001) and while going up and down stairs (Campbell and Buehler, 2003; Moore et al., 2002) (Figure 3). Finally, it was adopted by De Lasa and Buehler (2001) to analyze the performance of the SCOUT-II quadruped robot with reduced actuation.

Some authors argued that, contrary to the use of the mechanical power for computing the specific resistance (used in most applications), this index should be computed using the electrical power, in order to be able to consider several significant energy losses that occur (Campbell and Buehler, 2003; De Lasa and Buehler, 2001; Saranli et al., 2001). This allows an alternative definition for the specific resistance (Moore et al., 2002; Van der Linde, 1998), yielding:

$$
\rho=\frac{E}{M g d}
$$




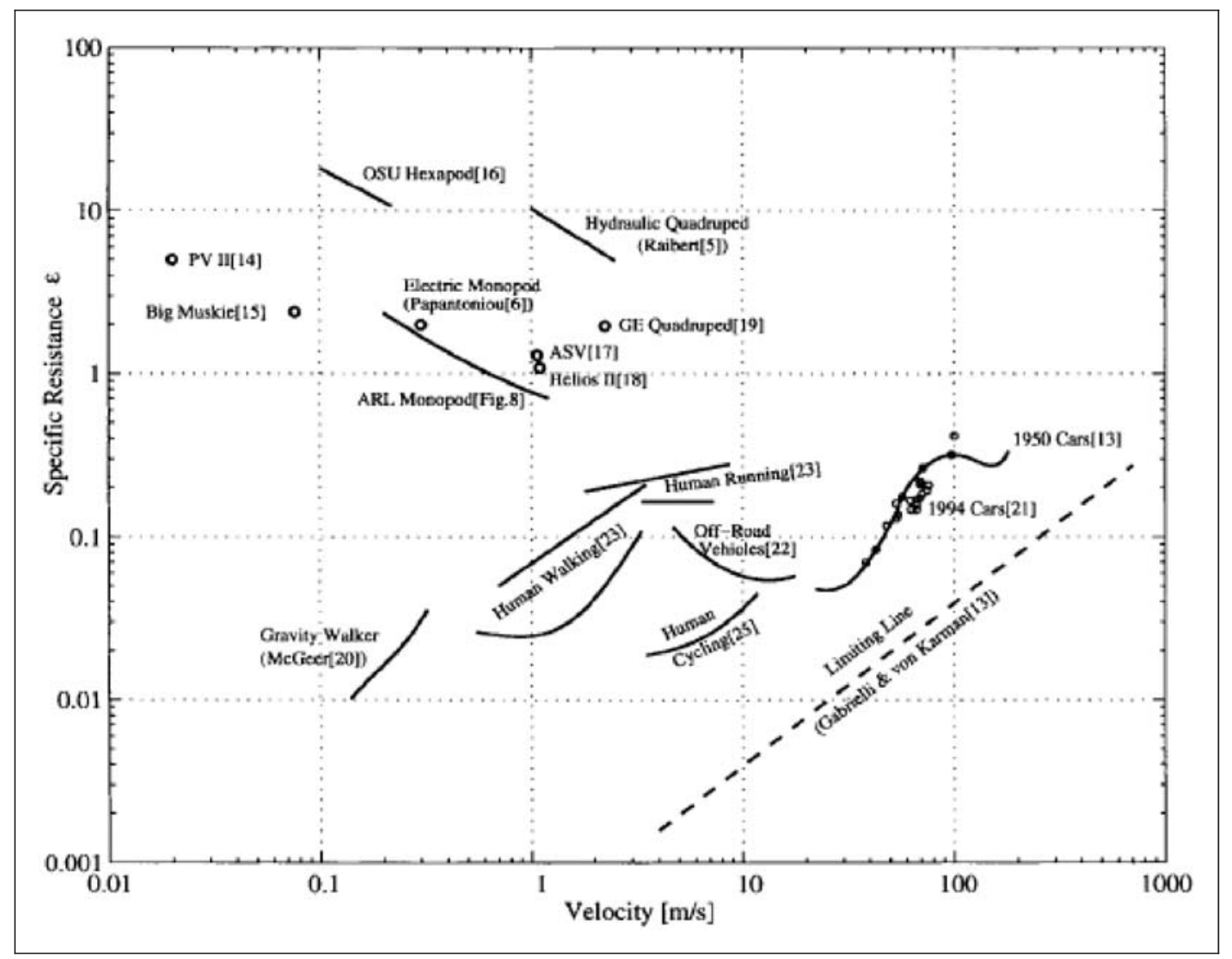

Figure 2. Specific resistance for selected land vehicles and human running. Reproduced with kind permission from the IEEE (Gregorio et al., 1997).

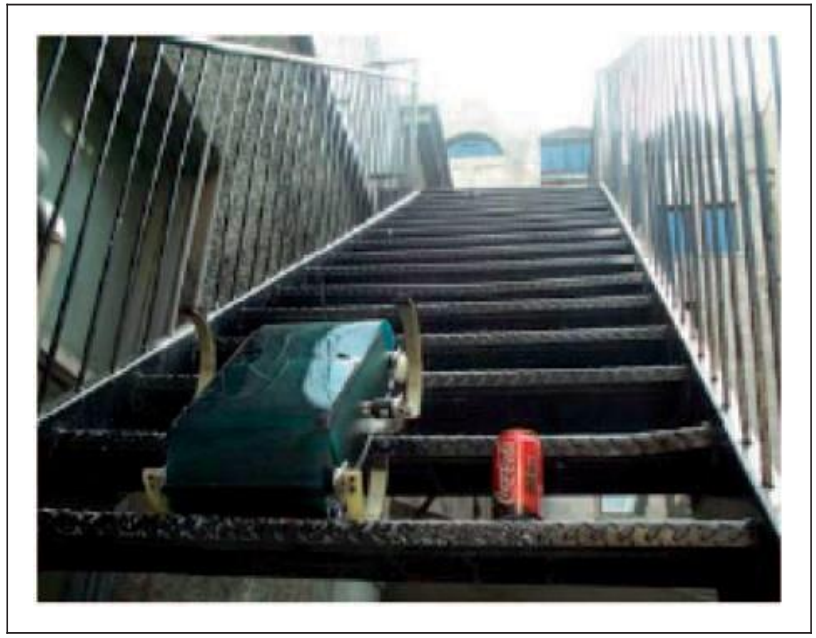

Figure 3. RHex hexapod robot climbing stairs. Reproduced with kind permission from the IEEE (Moore et al., 2002).

where $E$ represents the total electrical energy consumption for a linear displacement $d$.

To prove their proposal, De Lasa and Buehler (2001) computed the mechanical and the electrical power of the
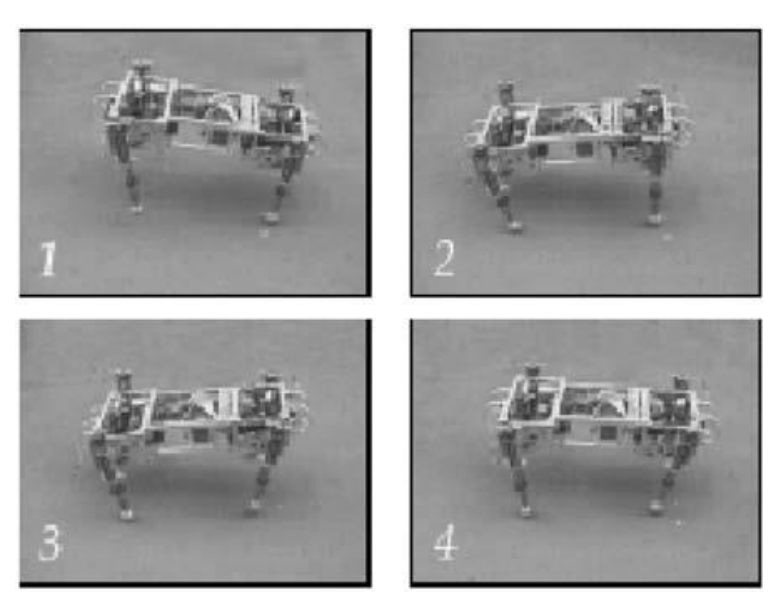

Figure 4. SCOUT-II quadruped robot in a bounding gait. Reproduced with kind permission from the IEEE (De Lasa and Buehler, 200I).

SCOUT-II robot (Figure 4), being the electrical power approximately ten times higher than the mechanical power. This result is also reflected in the specific resistance, that these authors determined using the two alternatives. 


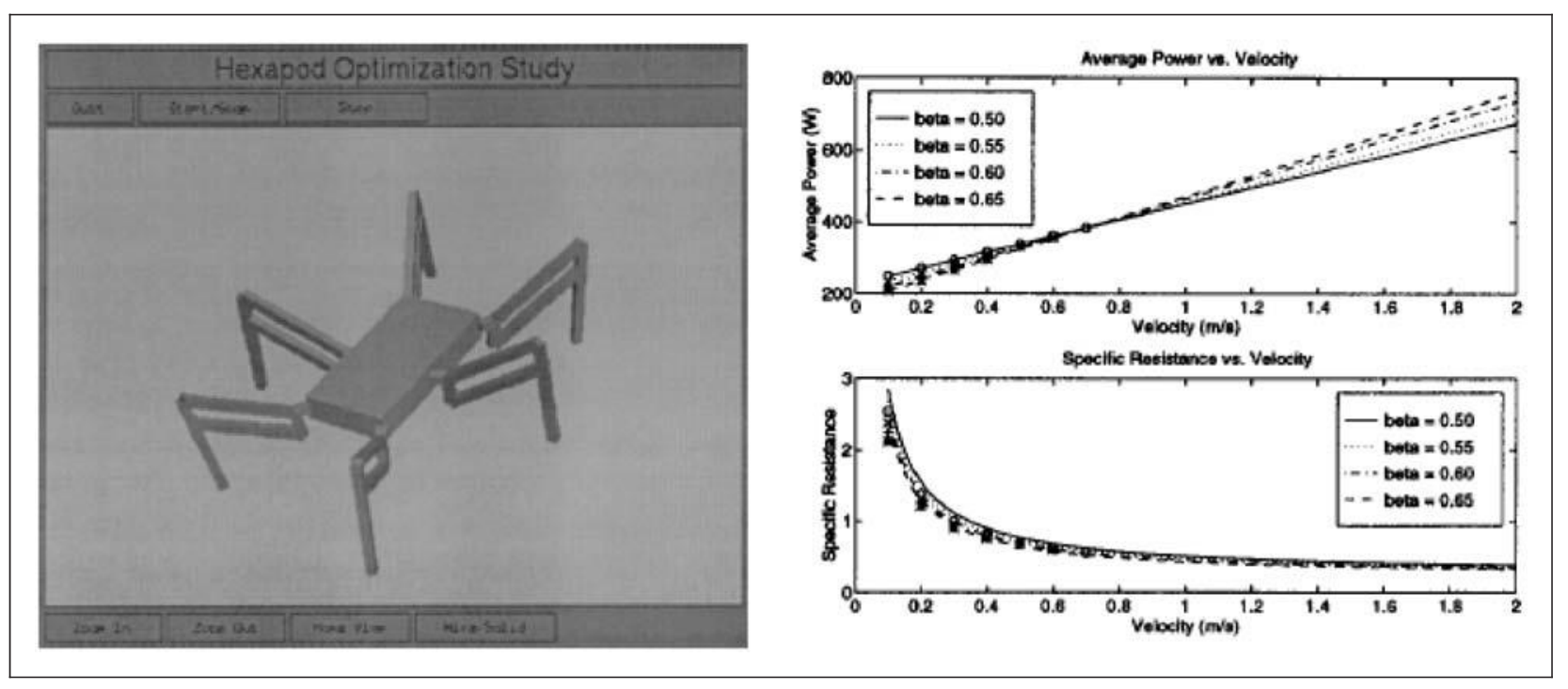

Figure 5. Insect-like hexapod robot simulation model (left) (Marhefka and Orin, 1998) and average power and specific resistance vs. velocity (right) (Marhefka and Orin, 1997). Reproduced with kind permission from the IEEE.

A slightly different use of this index is given by Lapshin (1995) that estimates the energy consumption of legged vehicles moving along straight trajectories, when using statically stable gaits. The results are compared with the energy consumptions of other vehicles and animals, based on the specific resistance index (1). In the analysis Lapshin makes use of simplified models of the energy consumption in legged robots. Lapshin considers that the energy consumption on these vehicles is divided into three independent parts, namely (i) to support the vehicle weight, (ii) to generate traction, and (iii) to make the legs oscillate in relation to the body. Lapshin concludes that the robot should walk with a reduced value of the duty factor, the leg should move with a step acceleration and deceleration during the transfer phase, the step length should be kept to a minimum and the body height should be maximum.

A similar approach is presented by Zhoga (1998) that analyzes the energy consumption of an eightlegged system, based in the dynamic model, and compares it with the energy consumption of machines with equivalent dimensions, but involving wheels or tracks for their locomotion. Zhoga also presents results of the variation of the energy consumption for distinct ground characteristics, curve radius and speeds.

Briskin et al. (2003) adopted the energy consumption, computed from the dynamic model, for the optimization and performance analysis of the Walking Chassis for Multipurposes robot.

Pedroche et al. (2003) go even further and present a detailed model of the energy consumption of legged robots based on the robot and actuators models (including the electrical and mechanical parts). These models are latter applied for analysing the influence of the leg configuration, of the body weight, of the foot trajectory and of the speed profile on the energy consumption of the SILO4 quadruped robot. More recently, Guardabrazo and de Santos (2004) apply an identical methodology to a biped robot.

Gregorio et al. (1997) considered the maximum torque needed for the hip motor of a monopod robot as a way to design the transmission parameters of this joint. In a second phase, they used three indices, namely the absolute values of the instantaneous mechanical power on the hip joint of the monopod robot, its integral over time, and the specific resistance index, to analyze the robot performance when moving at different speeds, and when adopting different hip trajectories. These authors compared also the specific resistance of their robot, with those of other legged, wheeled and tracked vehicles and living beings.

Marhefka and Orin (1997) simulated an hexapod robot with an insect-like structure and three DOF legs (see Figure 5, left), moving with a statically stable gait, in order to study how the locomotion parameters affect the energy consumption. They used as optimization criterion the minimization of the consumed power along a complete locomotion cycle (see Figure 5, right). These researchers presented a set of rules to establish the speed, the places over the ground for the feet placement, the body height, the duty factor and the step length, to apply during the gait generation in order to minimize the required energy. Later, Marhefka and Orin (1998) presented an extension of their work using as optimization criterion the minimization of the power supplied to each of the joint actuators, 
making use of the optimization of the feet forces. In this study, were presented different methodologies to optimize the force distribution by the robot legs and the results werecompared.

Ahmadi and Buehler (1999) considered the integral of the absolute value of the instantaneous 'shaft power' $(E)$ of a monopod robot to analyze its performance, according with the expression:

$$
E=\int_{t_{0}}^{t_{1}}|\tau \omega| d t
$$

where $r$ and ! are the torque and angular velocity of the motor, respectively.

Zielin' ska (2000) and Zielin'ska, et al. (2001)

$$
\text { deter- }
$$

mined the optimal design parameters of an hexapod robot (lengths of the legs links, body dimensions and leg structure) and of its actuators (maximum torque and gear ratio). The objective function consisted in the minimization of the energy consumption of the robot joint motors, estimated from the reaction forces foreseen on the robot feet.

Silva and Machado (2008) studied the locomotion parameters of an hexapod robot using four performance measures computed through the dynamic model of the studied mechanism.

The key measure in their analysis was the mean absolute density of energy per travelled distance $E_{a v}$ that is computed by taking the absolute value of the mechanical power. A Considering that the robot has $n$ legs and that each robot leg has $m$ joints, the mechanical power is the product of the actuator torque and angular velocity. The value of $E_{a v}$ is then obtained by averaging the mechanical absolute energy delivered over the travelled distance $d$ :

$$
E_{a v}=\frac{1}{d} \sum_{i=1}^{n} \sum_{j=1}^{m} \int_{0}^{T}\left|\tau_{i j}(t) \dot{\theta}_{i j}(t)\right| d t \quad\left[\mathrm{Jm}^{-1}\right]
$$

where $r_{i j}$ represents the motor torque and $8_{i j}$ the angu-

lar velocity, for the $i^{\text {th }}$ leg and $j^{\text {th }}$ joint.

Minimising the power appears to be an important issue, but it may occur for short time periods, very high power demands. In such cases, the average value can be small but the peaks are not achievable. Therefore, an alternative index proposed by the authors was the standard deviation per meter that evaluates the power variability (or dispersion) over a complete cycle $T$ and travelled distance $d$ :

$$
D_{a v}=\frac{1}{d} \sqrt{\frac{1}{T} \int_{0}^{T}\left[\sum_{i=1}^{n} \sum_{j=1}^{m}\left|\tau_{i j}(t) \dot{\theta}_{i j}(t)\right|-\frac{E_{a v}}{T}\right]^{2}} d t \quad\left[\mathrm{Wm}^{-1}\right]
$$

Another alternative optimisation strategy for an actuated system consists on $T_{L}$, the density of power lost in the joint actuators per travelled distance $d$, defined as:

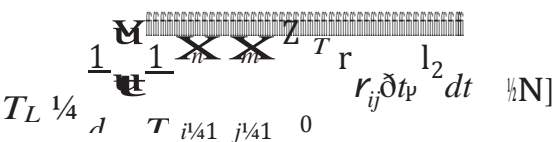

A fourth possible optimisation strategy considers the forces that occur on the hips of the robot per travelled distance $d$ and time spent on the travel $T$, giving rise to the index mean force on the hips per meter defined as:

$$
\text { 1 } F_{L}=\frac{1}{d} \sqrt{\frac{1}{T} \sum_{i=1}^{n} \int_{0}^{T}\left\{\left[f_{i x}(t)\right]^{2}+\left[f_{i j}(t)\right]^{2}\right\} d t}\left[\mathrm{Nm}^{-1}\right]
$$

In this equation, $f_{i x}$ and $f_{i y}$ represent the forces that act on the hip of the $i^{\text {th }}$ leg of the robot along the $x$ and $y$ directions, respectively.

A good system performance requires the simultaneous minimization of all indices $\left\{E_{a v}, D_{a v}, T_{L}\right.$, $\left.F_{L}\right\}$.

Wollherr et al. (2002) developed numerical simulations, based on the complete robot dynamical model, and adopted the minimization of two indices for the design of a small humanoid robot with 17 DOF. The main goal of these authors was the selection of motors and gears to use in the robot joints. One of the indices requires the minimization of the square of the torques and the other addresses the total amount of the mechanical power in the robot joints, according with:

$$
\min _{\tau}\left\{\int_{0}^{t_{f}}{ }^{T} \tau d t\right\}
$$

where $r$ is the input torque, while assuring that the maximum power consumption $\mathrm{M}_{W}$ should comply to the restriction:

$$
t \in\left[0, t_{f}\right], \max _{i \in\{1, \ldots, m\}}\left\{\left|\dot{\theta}_{i}(t) \tau_{i}(t)\right| \leq M_{W}\right\}
$$

being 8 the joint angular velocity and $m$ is the total number of joints. For the different models under study, the optimal control problem during one robot step was solved numerically to obtain the joint trajectories with the solutions in terms of speed and acceleration.

\section{Less used approaches to the optimization of legged robots}

Besides the criteria for the legged robots optimization mentioned in the previous sections, other approaches have also been sporadically used. 
Koyachi et al. (1997) developed the hexapod MELMANTIS-1 whose legs can also function as manipulators. For the design of this robot, and for its evolution named MELMANTIS-2, they studied the

variation of the manipulability ellipsoids (Yoshikawa, 1990) with a set of the robot leg/arm mechanism parameters.

The manipulability ellipsoids have also been used for the development of the MEL HORSE II. This robot, whose constructive and working principle is based on the horse and on the dinosaur, may behave as a biped or as a quadruped. The front and rear legs present different characteristics and Takeuchi (1999) used a design index based on the Yoshikawa (1990) manipulability concept.

Kang et al. (2003) also adopted the manipulability concept (Yoshikawa, 1990) during the design of the MRWALLSPECT-III climbing robot, as an optimization function to determine the optimal length of the legs links.

Silva and Machado (1997) for a biped, and Silva and Machado (2008) for an hexapod, used an approach based on the concepts of manipulability (Yoshikawa, 1990), called the locomobility analysis, to study the optimum locomotion parameters for a multi-legged robot.

The analysis is based on two indices obtained by averaging the distance among the centre of the ellipsoids and its intersections with the tangent to the desired trajectories, either of the foot $\left(E_{F}\right)$ or of the robot body $\left(E_{B}\right)$, over a complete locomotion cycle $T$, originating the foot $\left(L_{F}\right)$ and body $\left(L_{B}\right)$ locomobility indices:

$$
\begin{aligned}
L_{F} & =\frac{1}{T} \int_{0}^{T} E_{F}(t) d t \quad[\mathrm{~m}] \\
L_{B} & =\frac{1}{T} \int_{0}^{T} E_{B}(t) d t \quad[\mathrm{~m}]
\end{aligned}
$$

The most suitable trajectory is the one that maximises $L_{F}$ and $L_{B}$. These authors proposed also the 'perturbation analysis' for determining how the robot model stands with trajectory variations. For this purpose, the joint trajectories are first computed by the inverse kinematics algorithm. Afterwards, the joint angular acceleration vectors are 'corrupted' by small amplitude additive noise (according to an uniform distribution, with zero mean). As a result, the joint trajectories of the legs (and the robot feet cartesian trajectories, considering that the hips follow the right trajectory) suffer some distortion. By computing the mechanism forward kinematics, are determined two indices based on the statistical average of the mean square error:

$$
\begin{aligned}
& \xi_{\dot{x}}=\frac{1}{N_{S}} \sum_{i=1}^{N_{S}} \sqrt{\frac{1}{T} \frac{1}{n} \sum_{i=1}^{n} \int_{0}^{T}\left[\dot{x}_{i F n}(t)-\dot{x}_{i F d}(t)\right]^{2} d t}\left[\mathrm{~ms}^{-1}\right] \\
& \xi_{\dot{y}}=\frac{1}{N_{S}} \sum_{i=1}^{N_{S}} \sqrt{\frac{11}{T} \frac{1}{n} \sum_{i=1}^{n} \int_{0}^{T}\left[\dot{y}_{i F n}(t)-\dot{y}_{i F d}(t)\right]^{2} d t}\left[\mathrm{~ms}^{-1}\right]
\end{aligned}
$$

where $\mathrm{N}_{S}$ is the total number of steps for averaging purposes, $x_{j F d}$ and $x_{-i F n}$ are the $i^{\text {th }}$ samples of the horizontal velocities, desired and 'corrupted' with noise, on the robot foot, respectively, $y_{j F d}$ and $y_{j F n}$ are the $i^{\text {th }}$ samples of the vertical velocities, desired and 'corrupted' with noise, on the robot foot, respectively. In these equations, the index $i$ corresponds to the leg number, the index $F$ means that the variables correspond to the foot trajectory and the indices $d$ and $n$ are related to the desired trajectory and the trajectory corrupted with noise, respectively. The stochastic perturbation penalises the system's performance and the objective is the minimization of both indices $f_{x}$ and $f_{y_{-}}$.

Van den Doel and Pai (1997) computed the optimal modes of locomotion of the Platonic Beast walking robot, on different slopes and for several designs. They adopted two performance measures, the first proportional to the total motion of all joints during the robot locomotion (that the authors assume as being a rough approximation to the robot energy consumption) and the second determining the maximal speed of locomotion, for given limits of the joint speeds. Their results indicate that a variable link length can improve the ability of the robot to walk over steep slopes.

Shieh et al. (1997) used a multiobjective optimization scheme, based on the feasible sequential quadratic programming technique, for the design of a walking machine equipped with a one DOF leg mechanism. Two design objectives were simultaneous considered in the model: to minimize the peak crank torque, for the entire foot-point path, and the leg size at a prescribed leg posture. The algorithm also included constraint functions, namely concerning the stride length, the foot-path height, the four-bar transmission angle, the skew-pantograph transmission angle and the orientation angle of the lowest link. The optimization results showed a dramatic reduction in the mechanism size and a moderate reduction in the actuating torque from its preliminary design.

Shoval et al. (1999) developed a quadruped robot having four DOF legs. This robot has the objective of moving inside pipes and tunnels, whose walls have a very low coefficient of friction. For the robot design, 
namely for determining the body width, the total length of each of its legs and the total radius, they adopted geometric considerations. Among them, these research-

ers considered the requirements (i) that the central dimension of the robot body should be less than the minor dimension of the tunnel where the robot would move, and (ii) that the width of the robot body plus

twice the legs length should be larger than the maximum distance among the tunnel walls, to allow the robot reaching the tunnel walls with its legs. The robot radius was selected in a way that the reachable spots of the robot feet could form a continuous area along the tunnel in which the robot is required to move.

Kim et al. (2000) proposed the use of the following index to optimize the lengths of the links of a biped robot structure:

$$
P=\frac{\sqrt{\int_{0}^{T} \sum \tau_{G}^{2} d t}}{d}, \sum \tau_{G}^{2}=\tau_{G_{1}}^{2}+\tau_{G_{2}}^{2}+\tau_{G_{3}}^{2}+\tau_{G_{4}}^{2}
$$

where $T$ is the single support period and ${ }^{\mathrm{P}} r_{G}^{2}$ is the sum of the squares of the joint actuators static torques. The numerator of this index measures the static torque required during the single support phase and the denominator is the travelled distance along one step. Therefore, the criteria may be interpreted as the static torque needed by travelled distance.

Another strategy for artificial locomotion systems optimization was applied by Liu and Howard (2003) to crab-like robots, based on their kinematic work volume. On their study were analyzed the different alternative kinematic configurations of the robot legs, the corresponding restrictions, and were identified the best configurations (and the corresponding geometric parameters) that could maximize the kinematic work volume.

Another relevant problem in walking robots, that is often the target of optimization, is the aspect of the force distribution in the robot legs (or feet) (Chen et al., 1998). A reasonable basis for allocation of contact forces among the feet is that the tendency of each foot to slip should be minimized. Since the effective coefficient of friction between foot and ground is never accurately known, the logical strategy is to attempt to minimize the maximum of the angles between the contact forces and the normals to the surface at the contact points (Waldron, 1997). This problem is more acute in situations on which the ground surface is soft (Gorinevsky and Shneider, 1991) or the terrain is rough (Gardner, 1991).

Gorinevsky and Shneider (1991) developed and tested experimentally algorithms for the control of the foot-force distribution on hexapods, when they are walking over rigid and elastically deformable surfaces, or over surfaces subjected to irreversible deformation. On the other hand, Gardner (1991) used three techniques to solve the force distribution problem, and concluded that the optimal force distribution possessed the characteristic that all of the force ratios at the feet are identical. Based on this observation, presented a method that achieves good performance from the standpoint of traction and can be computed in a fraction of the time.

Marhefka and Orin (1998) presented different methodologies to optimize the force distribution by the robot legs, in order to minimize the power supplied to each of the joint actuators, and the results were compared.

\section{Conclusions}

During recent years the interest in robotic systems able to move by means similar to those found in nature has grown steadily. Legged robots present significant advantages over wheeled and tracked vehicles because they allow locomotion in terrain inaccessible to these traditional vehicles, since they do not need a continuous support surface.

Although the referred aspects indicate that legged locomotion is advantageous when compared with traditional locomotion systems, it should be kept in mind that, in their present state of development, these vehicles still suffer from huge limitations, since they exhibit low speeds, are difficult to build and need complex control algorithms. Besides, today's mechanisms are heavy, since they need a large number of actuators to move multiple DOF legs, to which one should add large energy consumption.

It is expected that in the future vehicles adopting locomotion through artificial legs may become an efficient transportation mode, that can compete with other classical transportation means. However, as stated previously, in their present state of development, there are several aspects that have to be improved and optimized.

One of the major drawbacks of this technology is its higher energy consumption, when compared with traditional forms of locomotion. It is verified that the

locomotion efficiency of the existing robots is very low when compared with living animals, or wheeled vehicles.

Therefore, a deeper attention should be devoted to the optimization of such systems, and it is verified that different optimization strategies have been proposed and applied to these systems either during its design and construction, or during its operation, namely in the gait selection and on its adaptation to the terrain and locomotion conditions.

This paper has presented a survey of several strategies, namely the mechatronic mimic of biological 
animals characteristics, the use of evolutionary computation for the optimization of the legged structure parameters, the adoption of good mechanical project rules, the optimization of power and energy-based indices and other complementary approaches.

\section{Acknowledgements}

The authors would like to acknowledge the anonymous reviewers whose valuable comments have allowed the improvement of this paper.

\section{References}

Ahmadi M and Buehler M (1999) The ARL monopod II running robot: control and energetics, in Proceedings of the 1999 IEEE International Conference on Robotics and Automation, Detroit, MI, May 10-15, pp.1689-1694.

Albert A and Gerth W (2001) New path planning algorithms for higher gait stability of a bipedal robot. In: Berns $\mathrm{K}$ and Dillmann (eds) CLAWAR 2001 - Fourth International Conference on Climbing and Walking Robots - From Biology to Industrial Applications, Bury St. Edmunds and London, UK:

Professional Engineering Publications Ltd. Albiez J and Berns K (2005) Biological inspired walking - how much nature do we need? In: Armada MA and de Santos Gonza' lez (eds) Climbing and Walking Robots. Berlin Heidelberg, Germany: Springer-Verlag.

Alexander RMcN (1984) The gaits of bipedal and quadrupedal animals. The International Journal of Robotics Research 3(2): 49-59.

Alexander RMcN (1990) Three uses for springs in legged locomotion. The International Journal of Robotics Research 9(2): 53-61.

Alexander RMcN (2005) Problems of scale for walking and climbing animals. In: Armada MA and Gonza' lez de Santos P (eds) Climbing and Walking Robots. Berlin Heidelberg, Germany: Springer-Verlag.

Ayers J (2004) Underwater walking. Arthropod Structure \& Development 33(3):347-360.

Bekker MG (1960) Off-The-Road Locomotion. Ann Arbor, MI: University of Michigan Press.

Bekker MG (1969) Introduction to Terrain Vehicle Systems. Ann Arbor, MI: University of Michigan Press.

Breithaupt R, Zahedi K, Hertzberg J, Pasemann F and Dahnke J (2002) Robo-Salamander - an approach for the benefit of both robotics and biology. In: Bidaud $\mathrm{P}$ and Amar FB (eds) Climbing and Walking Robots - Fifth International Conference on Climbing and Walking Robots and the Support Technologies for Mobile Machines. Bury St. Edmunds and London, UK: Professional Engineering Publications Ltd.

Briskin ES, Chernyshev VV and Maloletov AV (2003) On conception of walking machines designing, in Proceedings of the ICAR 2003 - 11th International Conference on Advanced Robotics, 30 June-3 July, Coimbra, Portugal, pp. 1763-1768.

Buehler M (2001) RePaC design and control - cheap and fast autonomous runners. In: Berns K and Dillmann R (eds) CLAWAR 2001 - 4th International Conference on Climbing and Walking Robots - From Biology to Industrial Applications. Bury St. Edmunds and London, UK: Professional Engineering Publications Ltd.

Buehler M, Battaglia R, Cocosco A, Hawker G, Sarkis J and Yamazaki K (1998) SCOUT: A simple quadruped that walks, climbs and runs. In Proceedings of the 1998 IEEE International Conference on Robotics and Automation, 1620 May, Leuven, Belgium, pp. 1707-1712.

Buehler M, Cocosco A, Yamazaki K and Battaglia R (1999) Stable open loop walking in quadruped robots with stick legs, in Proceedings of the 1999 IEEE International Conference on Robotics and Automation, 10-15 May, Detroit, MI, pp.2348-2353.

Caldwell DG, Medrano-Cerda GA and Bowler CJ (1997) Investigation of bipedal robot locomotion using pneumatic muscle actuators, in Proceedings of the 1997 IEEE International Conference on Robotics and Automation, 2025 April 1997, Albuquerque, NM, pp. 799-804.

Campbell D and Buehler M (2003) Stair descent in the simple hexapod 'RHex', in Proceedings of the 2003 IEEE International Conference on Robotics and Automation, 1419 September, Taipei, Taiwan, pp. 1380-1385.

Cham JG, Bailey SA, Clark JE, Full RJ and Cutkosky MR (2002) Fast and robust: hexapedal robots via shape deposition manufacturing. International Journal of Robotics Research 21(10-11): 869-882.

Chen J-S, Cheng F-T, Yang K-T, Kung F-C and Sun Y-Y (1998) Solving the optimal force distribution problem in multilegged vehicles, in Proceedings of the 1998 IEEE International Conference on Robotics and Automation, 16-20 May, Leuven, Belgium, pp.471-476.

De Lasa M and Buehler M (2001) Dynamic compliant quadruped walking, in Proceedings of the 2001 IEEE International Conference on Robotics and Automation, 2126 May 2001, Seoul, Korea, pp. 3153-3158.

Dunn ER and Howe RD (1996) Foot placement and velocity control in smooth bipedal walking, in Proceedings of the 1996 IEEE International Conference on Robotics and Automation, 22-28 April, Minneapolis, MN, pp. 578-583. Endo K and Maeno T (2003) Co-evolution of morphology and walking pattern of biped humanoid robot using evo-

lutionary computation - designing the real robot, in Proceedings of the 2003 IEEE International Conference on Robotics and Automation, 14-19 September, Taipei, Taiwan, pp. $1362-1367$.

Endo K, Maeno T and Kitano H (2002a) Co-evolution of morphology and walking pattern of biped humanoid robot using evolutionary computation - consideration of characteristic of the servomotors, in Proceedings of the 2002 IEEE/RSJ International Conference on Intelligent Robots and Systems, 30 September-5 October 2002, Lausanne, Switzerland, pp. 2678-2683.

Endo K, Yamasaki F, Maeno T and Kitano H (2002b) a method for co-evolving morphology and walking pattern of biped humanoid robot, in Proceedings of the 2002 IEEE International Conference on Robotics and Automation, 1115 May 2002, Washington, DC, pp. 2775-2780.

Endo K, Maeno T and Kitano H (2003) Co-evolution of morphology and walking pattern of biped humanoid robot using evolutionary computation - evolutionary 
designing method and its evaluation, in Proceedings of the 2003 IEEE/RSJ International Conference on Intelligent Robots and Systems, 27-31 October, Las Vegas, NV, pp. $340-345$.

Espenschied KS, Quinn RD, Chiel HJ and Beer RD (1996) Biologically-based distributed control and local reflexes improve rough terrain locomotion in a hexapod robot. Robotics and Autonomous Systems 18: 59-64.

Espiau B and Sardain P (2000) The anthropomorphic biped robot BIP2000, in Proceedings of the 2000 IEEE International Conference on Robotics and Automation, 2428 April, San Francisco, CA, pp. 3997-4002.

Espiau B and The BIP Team (1997) BIP: a joint project for the development of an anthropomorphic biped robot, in Proceedings of ICAR'97 - Eighth International Conference on Advanced Robotics, 7-9 July, Monterey, CA, pp. 267272.

Estremera J, Garcia E and Gonzalez de Santos P (2003) A continuous free crab gait for quadruped robots on irregular terrain. In: Muscato G and Longo D (eds) Climbing and Walking Robots - Sixth International Conference on Climbing and Walking Robots and the Support Technologies for Mobile Machines. Bury St. Edmunds and London, UK: Professional Engineering Publishing Ltd.

Farritor S, Dubowsky S, Rutman N and Cole J (1996) A systems-level modular design approach to field robotics, in Proceedings of the 1996 IEEE International Conference on Robotics and Automation, 22-28 April, Minneapolis, MN, pp. 2890-2895.

Gabrielli G and Von Ka' rma' n T (1950) What price speed? specific power required for propulsion of vehicles. Mechanical Engineering, ASME 72(10): 775-781.

Garcia E, Estremera J and Gonzalez de Santos P (2002) A classification of stability margins for walking robots. In: Bidaud $\mathrm{P}$ and Amar FB (eds) Climbing and Walking Robots - Fifth International Conference on Climbing and Walking Robots and the Support Technologies for Mobile Machines. Bury St. Edmunds and London, UK: Professional Engineering Publications Ltd.

Gardner JF (1991) Characteristics and approximations of optimal force distributions in walking machines on rough terrain, in Proceedings of ICAR'91 - Fifth International Conference on Advanced Robotics, 19-22, June 1991, Pisa, Italy, pp. 613-618.

Genta G and Amati N (2001) Planar motion hexapod walking machines - a new configuration. In: Berns $\mathrm{K}$ and Dillmann R (eds) CLAWAR 2001 - Fourth International Conference on Climbing and Walking Robots - From Biology to Industrial Applications. Bury St. Edmunds and London, UK: Professional Engineering Publications Ltd.

Gorinevsky DM and Shneider AY (1991) Force control in locomotion of legged vehicle over rigid and soft surfaces, in Proceedings of ICAR'91 - Fifth International Conference on Advanced Robotics, 19-22 June 1991, Pisa, Italy, pp. 609-612.

Gravez F, Mohamed B and Ouezdou FB (2002) Dynamic simulation of a humanoid robot with four DOFs torso, in Proceedings of the 2002 IEEE International Conference on Robotics and Automation, 11-15 May 2002, Washington, DC, pp. 511-516.
Gregorio P, Ahmadi M and Buehler M (1997) Design, control, and energetics of an electrically actuated legged robot. IEEE Transactions on Systems, Man and Cybernetics Part B: Cybernetics 27(4): 626-634.

Guardabrazo TA and de Santos PG (2004) Building an energetic model to evaluate and optimize power consumption in walking robots. Industrial Robot 31(2): 201-208.

Habumuremyi J-C and Doroftei I (2001) Mechanical design and MANFIS control of a leg for a new demining walking robot. In: Berns K and Dillmann $\mathrm{R}$ (eds) CLAWAR 2001 - Fourth International Conference on Climbing and Walking Robots - From Biology to Industrial Applications. Bury St. Edmunds and London, UK: Professional Engineering Publications Ltd.

Hardt M and von Stryk O (2002) Increasing stability in dynamic gaits using numerical optimization, in Proceedings of the 15th Triennial IFAC World Congress, 21-26 July 2002, Barcelona, Spain, in CD-ROM.

Hawker G and Buehler M (2000) Quadruped trotting with passive knees - design, control, and experiments. In Proceedings of the 2000 IEEE International Conference on Robotics and Automation, 24-28 April, San Francisco, CA, pp. 3046-3051.

Hennion B, Pill J and Guinot J-C (2005) A biologically inspired model for quadruped locomotion. In: Tokhi MO, Virk GS and Hossain MA (eds) Climbing and Walking Robots. Berlin Heidelberg: Germany: SpringerVerlag, 49-56.

Hirai K, Hirose M, Haikawa Y and Takenaka T (1998) The development of Honda humanoid robot, in Proceedings of the 1998 IEEE International Conference on Robotics and Automation, 16-20 May, Leuven, Belgium, pp. 1321-1326.

Hirose S and Arikawa K (2000) Coupled and decoupled actuation of robotic mechanisms, in Proceedings of the 2000 IEEE International Conference on Robotics and Automation, 24-28 April, San Francisco, CA, pp. 33-39.

Hirose S, Yoneda K and Tsukagoshi H (1997) TITAN VII: quadruped walking and manipulating robot on a steep slope, in Proceedings of the 1997 IEEE International Conference on Robotics and Automation, 20-25 April 1997, Albuquerque, NM, pp. 494-500.

Hornby GS, Takamura, S, Yokono J, Hanagata O, Yamamoto T and Fujita M (2000) Evolving robust gaits with AIBO, in Proceedings of the 2000 IEEE International Conference on Robotics and Automation, 24-28 April, San Francisco, CA, pp.3040-3045.

Hornby GS, Lipson H and Pollack JB (2001) Evolution of generative design systems for modular physical robots, in Proceedings of the 2001 IEEE International Conference on Robotics and Automation, 21-26 May 2001, Seoul, Korea, pp. 4146-4151.

Huang MZ and Waldron KJ (1990) Relation between payload and speed in legged locomotion systems. IEEE Transactions on Robotics and Automation 6(5): 570-577.

Hugel V and Blazevic P (1999) Towards efficient implementation of quadruped gaits with duty factor of 0.75 , in Proceedings of the 1999 IEEE International Conference on Robotics and Automation, 10-15 May, Detroit, MI, pp. 2360-2365. 
Hugel V, Bonnin P and Blazevic P (2000) Speed control for quadruped using duty factor, in Proceedings of the 2000 IEEE/RSJ International Conference on Intelligent Robots and Systems, 31 October-5 November 2000, Takamatsu, Japan, pp. 985-990.

Ihme T and Deutscher M (2001) Design and control aspects for six-legged walking robots to realize adaptation to the environment. In: Berns $\mathrm{K}$ and Dillmann $\mathrm{R}$ (eds) CLAWAR 2001 - 4th International Conference on Climbing and Walking Robots - From Biology to Industrial Applications. Bury St. Edmunds and London, UK: Professional Engineering Publications Ltd.

Ishiguro A, Kawasumi $\mathrm{K}$ and Fujii A (2002) Increasing evolvability of a locomotion controller using a passivedynamic-walking embodiment, in Proceedings of the 2002 IEEE/RSJ International Conference on Intelligent Robots and Systems, 30 September-5 October 2002, Lausanne, Switzerland, pp. 2581-2586.

Jua' rez-Guerrero J, Mun oz-Gutie' rrez $\mathrm{S}$ and Cuevas WWM (1998) Design of a walking machine structure using evo- lutionary strategies. In Proceedings of the 1998 IEEE International Conference on Systems, Man and Cybernetics, 11-14 October 1998, San Diego, CA, pp. 1427-1432.

Kajita S, Nagasaki T, Yokoi K, Kaneko K and Tanie K (2002) Running pattern generation for a humanoid robot, in Proceedings of the 2002 IEEE International Conference on Robotics and Automation, 11-15 May 2002, Washington, DC, pp. 2755-2761.

Kaneko M, Mizuno A and Harada K (2002) Torque distribution for achieving a hugging walk, in Proceedings of the 2002 IEEE/RSJ International Conference on Intelligent Robots and Systems, 30 September-5 October 2002, Lausanne, Switzerland, pp. 2613-2618.

Kang T, Kim H, Son T and Choi H (2003) Design of quadruped walking and climbing robot, in Proceedings of the 2003 IEEE/RSJ International Conference on Intelligent Robots and Systems, 27-31 October 2003, Las Vegas, NV, pp. 619-624.

Kim S-H, Oh J-H and Lee K-H (2000) Design of 4 joints 3 links biped robot and its gaits, in Proceedings of the 2000 IEEE/RSJ International Conference on Intelligent Robots and Systems, 31 October-5 November 2000, Takamatsu, Japan, pp. 1155-1160.

Kohl N and Stone P (2004) Policy gradient reinforcement learning for fast quadrupedal locomotion, in Proceedings of the 2004 IEEE International Conference on Robotics and Automation, 26 April-1 May 2004, New Orleans, LA, pp. 2619-2624.

Koyachi N, Adachi H, Nakamura T and Nakano E (1991) Stair-climbing control of self-contained hexapod with semi-fixed gait, in Proceedings of ICAR'91 - Fifth International Conference on Advanced Robotics, 19-22 June 1991, Pisa, Italy, pp. 747-752.

Koyachi N, Arai T, Adachi H, Murakami A and Kawai K (1997) Mechanical design of hexapods with integrated limb mechanism: MELMANTIS-1 and MELMANTIS-2, in Proceedings of ICAR'97 - Eighth International Conference on Advanced Robotics, 7-9 July 1997, Monterey, CA, pp. 273-278.
Laksanacharoen S, Pollack AJ, Nelson GM, Quinn RD and Ritzmann RE (2000) Biomechanics and simulation of cricket for microrobot design, in Proceedings of the 2000 IEEE International Conference on Robotics and Automation, 24-28 April 2000, San Francisco, CA, pp. 1088-1094.

Lapshin VV (1995) Energy consumption of a walking machine. model estimations and optimization, in Proceedings of ICAR'95 - Seventh International Conference on Advanced Robotics, September 1995, Sant Feliu de Guixols, Catalonia, Spain, pp. 420-425.

Leger C (2000) DARWIN2K - An Evolutionary Approach to Automated Design for Robotics. Norwell, MA: Kluwer Academic Publishers.

Lipson H and Pollack JB (2000) Towards continuously reconfigurable self-designing robots, in Proceedings of the 2000 IEEE International Conference on Robotics and Automation, 24-28 April 2000, San Francisco, CA, pp. 1761-1766.

Liu A and Howard D (2003) The geometric design of crablike CLAWAR machines with good workspace characteristics. In: Muscato G and Longo D (eds) CLAWAR 2003 Sixth International Conference on Climbing and Walking Robots and the Support Technologies for Mobile Machines. Bury St. Edmunds and London, UK: Professional Engineering Publishing Ltd.

Lohmeier S, Lo“ ffler K, Gienger M, Ulbrich H and Pfeiffer F (2004) Computer system and control of biped "Johnnie", in Proceedings of the 2004 IEEE International Conference on Robotics and Automation, 26 April-1 May 2004, New Orleans, LA, pp. 4222-4227.

Marhefka DW and Orin DE (1997) Gait planning for energy efficiency in walking machines, in Proceedings of the 1997 IEEE International Conference on Robotics and Automation, 20-25 April 1997, Albuquerque, NM, pp. 474-480.

Marhefka DW and Orin DE (1998) Quadratic optimization of force distribution in walking machines, in Proceedings of the 1998 IEEE International Conference on Robotics and Automation, Leuven, Belgium, May 16-20, pp. 477-483.

Moore EZ, Campbell D, Grimminger F and Buehler M (2002) Reliable stair climbing in the simple hexapod 'RHex', in Proceedings of the 2002 IEEE International Conference on Robotics and Automation, 11-15 May 2002, Washington, DC, pp. 2222-2227.

Nelson GM and Quinn RD (1998) posture control of a cockroach-like robot, in Proceedings of the 1998 IEEE International Conference on Robotics and Automation, Leuven, Belgium, May 16-20, pp. 157-162.

Nelson GM, Quinn RD, Bachmann RJ, Flannigan WC, Ritzmann RE and Watson JT (1997) Design and simulation of a cockroach-like hexapod robot, in Proceedings of the 1997 IEEE International Conference on Robotics and Automation, 20-25 April 1997, Albuquerque, NM, pp. 1106-1111.

Neuhaus P and Kazerooni H (2000) Design and control of human assisted walking robot, in Proceedings of the 2000 IEEE International Conference on Robotics and Automation, 24-28 April 2000, San Francisco, CA, pp. 563-569. 
Nolfi S and Floreano D (2000) Evolutionary Robotics - The Biology, Intelligence, and Technology of Self-Organizing Machines. Cambridge, MA: The MIT Press.

Papadopoulos D and Buehler M (2000) Stable running in a quadruped robot with compliant legs, in Proceedings of the 2000 IEEE International Conference on Robotics and Automation, 24-28 April 2000, San Francisco, CA, pp. 444-449.

Pedroche TAG, Ruiz MAJ and de Santos PG (2003) A detailed power consumption model for walking robots. In: Muscato G and Longo D (eds) CLAWAR 2003 Sixth International Conference on Climbing and Walking Robots and the Support Technologies for Mobile Machines. Bury St. Edmunds and London, UK: Professional Engineering Publishing Ltd.

Pires EJS, Machado JAT and Oliveira PB de M (2001) An evolutionary approach to robot structure and trajectory optimization, in Proceedings of the ICAR'01 - Tenth International Conference on Advanced Robotics, 22-25 August 2001, Budapest, Hungary, pp. 333-338.

Quinn RD, Nelson GM, Bachmann RJ, Kingsley DA, Offi JT and Ritzmann RE (2001) Insect designs for improved mobility. In: Berns $\mathrm{K}$ and Dillmann R (eds) CLAWAR 2001 - 4th International Conference on Climbing and Walking Robots - From Biology to Industrial Applications. Bury St. Edmunds and London, UK: Professional Engineering Publications Ltd.

Quinn RD, Offi JT, Kingsley DA and Ritzmann RE (2002) Improved mobility through abstracted biological principles. In Proceedings of the 2002 IEEE/RSJ International Conference on Intelligent Robots and Systems, 30 September5 October 2002 Lausanne, Switzerland, pp. 2652-2657.

Raby EY and Orin DE (1999) Passive walking with leg compliance for energy efficient multilegged vehicles, in Proceedings of the 1999 IEEE International Conference on Robotics and Automation, Detroit, MI, May10-15, pp.1702-1707.

Reyes C and Gonzalez F (2005) Mechanical design optimization of a walking robot leg using genetic algorithm. In: Armada MA and de Santos Gonza' lez (eds) Climbing and Walking Robots. Berlin Heidelberg, Germany: Springer-Verlag.

Saranli U, Buehler M and Koditschek DE (2001) RHex - a simple and highly mobile hexapod robot. The International Journal of Robotics Research 20(7): 616-631.

Schneider A, Schmitz J and Cruse H (2005) A bio-inspired joint controller for the decentral control of a closed kinematic chain consisting of elastic joints, in Proceedings of 44th IEEE Conference on Decision and Control and the European Control Conference 2005, 12-15 December 2005, Seville, Spain, pp. 233-238.

Senta Y, Emura T, Noguchi Y, Hiraki M and Arakawa A (1995) Trotting gait of a quadruped robot using parallel crank-slider mechanism, in Proceedings of ICAR'95 Seventh International Conference on Advanced Robotics, September 1995, Sant Feliu de Guixols, Catalonia, Spain, pp. 406-411.

Shieh W-B, Tsai L-W and Azarm S (1997) Design and optimization of a one degree-of-freedom six-bar leg mechanism for a walking machine. Journal of Robotic Systems 14(12): 871-880.
Shoval S, Rimon E and Shapiro A (1999) Design of a spiderlike robot for motion with quasistatic force constraints, in Proceedings of the 1999 IEEE International Conference on Robotics and Automation, Detroit, MI, May 10-15, pp. 1377-1383.

Silva FM and Machado JAT (1997) Kinematic aspects of robotic biped locomotion systems, in Proceedings of the 1997 IEEE/RSJ International Conference on Intelligent Robots and Systems, 7-11 September 1997, Grenoble, France, pp. 266-271.

Silva MF and Machado JAT (2007) A historical perspective of legged robots. Journal of Vibration and Control 13(9-10): 1447-1486.

Silva MF and Machado JAT (2008) Kinematic and dynamic performance analysis of artificial legged systems. ROBOTICA 26(1): 19-39.

Silva MF and Machado JAT (2005) Energy efficiency of quadruped gaits. In: Tokhi MO, Virk GS and Hossain MA (eds) Climbing and Walking Robots. Berlin Heidelberg, Germany: Springer-Verlag.

Stelzer M, Hardt M and Von Stryk O (2003) Efficient dynamic modelling, numerical optimal control, and experimental results for various gaits of a quadruped robot. In: Muscato G and Longo D (eds) Climbing and Walking Robots - Sixth International Conference on Climbing and Walking Robots and the Support Technologies for Mobile Machines. Bury St. Edmunds and London, UK: Professional Engineering Publishing Ltd.

Takeuchi H (1999) Development of "MEL HORSE", in Proceedings of the 1999 IEEE International Conference on Robotics and Automation, 10-15 May, Detroit, MI, pp. 1057-1062.

Van den Doel K and Pai DK (1997) Performance measures for locomotion robots. Journal of Robotic Systems 14(2): 135-147.

Van der Linde RQ (1998) Active leg compliance for passive walking, in Proceedings of the 1998 IEEE International Conference on Robotics and Automation, 16-20 May, Leuven, Belgium pp. 2339-2344.

Waldron KJ (1997) Design of walking machines, in Proceedings of the 1997 IEEE International Conference on Robotics and Automation - Workshop 2: Recent Trends in Robot Locomotion, 20-25 April 1997, Albuquerque, NM.

Wang L, Xie M, Zhong ZW, Wang C and Zhang L (2008) Power analysis and structure optimization in the design of a humanoid robot. In: Marques L, de Almeida A, Tokhi MO and Virk GS (eds) Advances in Mobile Robots. London, UK: World Scientific.

Warren HA (2002) CLAWAR2 - mobile machines operating in outdoor unstructured terrains. In: Bidaud P and Amar FB (eds) Climbing and Walking Robots - Fifth International Conference on Climbing and Walking Robots and the Support Technologies for Mobile Machines. Bury St. Edmunds and London, UK: Professional Engineering Publications Ltd.

Weingarten JD, Lopes GAD, Buehler M, Groff RE and Kiditschek DE (2004) Automated gait adaptation for legged robots, in Proceedings of the 2004 IEEE International Conference on Robotics and Automation, 26 April-1 May 2004, New Orleans, LA, pp. 2153-2158. 
Witte H, Hackert R, Fischer MS, Ilg W, Albiez J, Dillmann R, et al. (2001a) Design criteria for the leg of a walking machine derived by biological inspiration from quadrupedal mammals. In: Berns Kand Dillmann R (eds) CLAWAR 2001 - 4th International Conference on Climbing and Walking Robots - From Biology to Industrial Applications. 24-26 September 2001, Bury St. Edmunds and London, UK: Professional Engineering Publications Ltd.

Witte H, Hackert R, Lilje KE, Schilling N, Voges D, Klauer $\mathrm{G}$, et al. (2001b) Transfer of biological principles into the construction of quadruped walking machines. In Proceedings of the Second International Workshop on Robot Motion and Control, Poznan', Poland, pp. 245-249.

Wollherr D, Hardt M, Buss M and von Stryk O (2002) Actuator selection and hardware realization of a small and fast-moving, autonomous humanoid robot, in Proceedings of the 2002 IEEE/RSJ International Conference on Intelligent Robots and Systems, Lausanne, Switzerland, pp. 2491-2496.

Yamaguchi J and Takanishi A (1998) Development of a leg part of a humanoid robot - design of a biped walking robot having antagonistic driven joints using a nonlinear spring mechanism. Advanced Robotics 11(6): 633-652.

Yoneda K (2001) Design of non-bio-mimetic walker with fewer actuators. In: Berns $\mathrm{K}$ and Dillmann R (eds)
CLAWAR 2001 - 4th International Conference on Climbing and Walking Robots - From Biology to Industrial Applications. Bury St. Edmunds and London, UK: Professional Engineering Publications Ltd.

Yoshikawa T (1990) Foundations of Robotics - Analysis and Control. Cambridge, MA: The MIT Press.

Zhoga VV (1998) Computation of walking robots movement energy expenditure, in Proceedings of the 1998 IEEE International Conference on Robotics and Automation, 1620 May, Leuven, Belgium, pp. 163-168.

Ziegler J and Banzhaf W (2001) Evolution of robot leg movements in a physical simulation. In: Berns K and Dillmann R (eds) CLAWAR 2001 - Fourth International Conference on Climbing and Walking Robots - From Biology to Industrial Applications. Bury St. Edmunds and London, UK: Professional Engineering Publications Ltd.

Zielin' ska T (2000) Efficiency analysis in the design of walking machines. Journal of Theoretical and Applied Mechanics 38(3): 693-705.

Zielin'ska T, Choong KC and Heng J (2001) Actuating system of six-legged walking machine. In: Berns $\mathrm{K}$ and Dillmann R (eds) CLAWAR 2001 - 4th International Conference on Climbing and Walking Robots - From Biology to Industrial Applications. Bury St. Edmunds and London, UK: Professional Engineering Publications Ltd. 